\title{
DEVELOPMENT OF A HYBRID RANS-LES MODEL BASED ON TEMPORAL FILTERING FOR NATURAL CONVECTION FLOWS
}

\author{
Puneeth Bikkanahally $^{1,2}$, Rémi Manceau ${ }^{1,3}$, Franck Mastrippolito ${ }^{1,4}$ \\ ${ }^{1}$ CNRS/Univ Pau \& Pays Adour/E2S UPPA, Laboratoire de Mathématiques et de leurs Applications \\ de Pau - Fédération IPRA, UMR5142, 64000 Pau, France \\ 2 pbmreddy@univ-pau.fr \\ 3 remi.manceau@univ-pau.fr \\ ${ }^{4}$ franck.mastrippolito@univ-pau.fr
}

Key Words: Natural convection, Hybrid RANS-LES, Turbulence modeling

Transient, buoyancy-affected flows play a major role in some industries, for instance in the design of nuclear power plants or the under-hood space of automobiles. In order to avoid expensive experiments and promote rapid design and development, it is quintessential to employ efficient, robust, and accurate CFD models to simulate such flows. With the aim of being cost-effective and retaining the ability to capture the necessary transient phenomena, the industry standard of using RANS models alone does not suffice, whereas LES is as of yet not affordable for industrial configurations. In this context, a Hybrid RANS-LES model based on temporal filtering, called Hybrid Temporal Large Eddy Simulation (HTLES) is developed, thus reducing computational costs by switching to RANS in the near-wall regions while being able to predict a large part of the turbulent scales in regions where it is necessary. The present work is based on the HTLES model developed for iso-thermal configurations [1], with the hybridisation applied to the standard $k-\omega$ SST model. The extension to natural convection flows is carried out using the DNS database of a differentially heated square cavity [2]. The test case is characterised by the three non-dimensional numbers viz. Rayleigh number $\left(R a=10^{11}\right)$, Prandtl number $(\operatorname{Pr}=0.71)$, and aspect $\operatorname{ratio}\left(A_{x}=L_{x} / L_{y}=1\right)$ and features a stratified core with a spatially developing buoyant boundary layer. The study reveals that the HTLES model is able to predict the mean profiles of temperature and velocity satisfactorily. Comparisons with URANS show that the HTLES model predicts the kinetic energy profiles more accurately, especially when considering coarser grids. However, it is noted that the current definition of the shielding function (used to enforce RANS close to the walls based on the Kolmogorov length scale) is inadvertently modifying the energy ratio far from the wall due to fluctuations in the dissipation rate, $\varepsilon$. This is further confirmed by way of a priori tests using the DNS database. It is essential to have a shielding function that is only effective in the near-wall regions. Therefore, to ward off this issue, a shielding function based on the Elliptic blending framework is developed. For the validation of the approach, flows in a differentially heated vertical channel [3] and a 4:1 cavity [4] are considered, in order to investigate the ability of the model to reproduce natural convection flows with very different stratification.

\section{REFERENCES}

[1] Duffal, V., de Laage de Meux, B., and Manceau, R. Development and Validation of a Hybrid RANS-LES Approach Based on Temporal Filtering. Volume 2: Computational Fluid Dynamics (July 
2019)

[2] Sebilleau, F. et al. Direct Numerical Simulation of an Air-Filled Differentially Heated Square Cavity With Rayleigh Numbers Up To $10^{11}$. International Journal of Heat and Mass Transfer (2018)

[3] Kiš, P., and Herwig, H. The near wall physics and wall functions for turbulent natural convection. International Journal of Heat and Mass Transfer (April 2012)

[4] Trias, F.X. et al. Direct numerical simulation of a differentially heated cavity of aspect ratio 4 with Rayleigh numbers up to $10^{11}$ - Part I: Numerical methods and time-averaged flow. International Journal of Heat and Mass Transfer (January 2010)
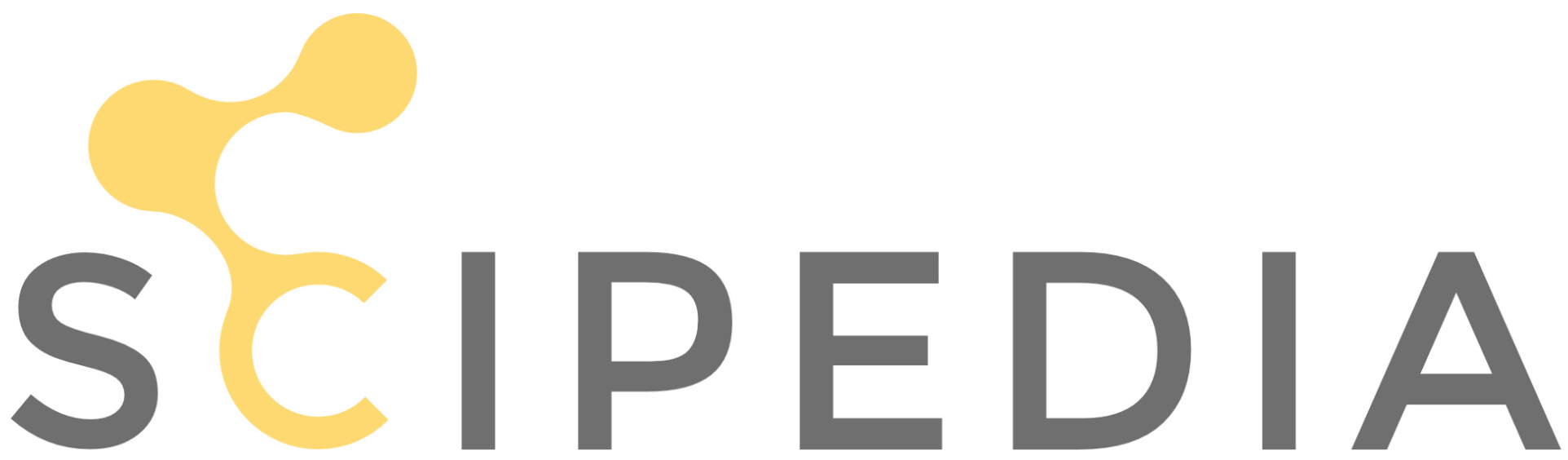

Register for free at https//www.scipedia.com to download the version without the watermark 